\title{
Long-term lipoprotein apheresis in the treatment of severe familial hypercholesterolemia refractory to high intensity statin therapy: Three year experience at a lipoprotein apheresis center
}

\author{
Agnieszka Mickiewicz ${ }^{1}$, Justyna Borowiec-Wolna ${ }^{1}$, Witold Bachorski ${ }^{1}$, \\ Natasza Gilis-Malinowska ${ }^{1}$, Rafał Gałąska ${ }^{1}$, Grzegorz Raczak ${ }^{2}$, \\ Magdalena Chmara ${ }^{3,4}$, Bartosz Wasąg ${ }^{3}$, Miłosz J. Jaguszewski ${ }^{1}$, \\ Marcin Fijałkowski ${ }^{1}$, Marcin Gruchała ${ }^{1}$ \\ ${ }^{1} 1^{\text {st }}$ Department of Cardiology, Medical University of Gdansk, Poland \\ ${ }^{2}$ Department of Cardiology and Electrotherapy, Medical University of Gdansk, Poland \\ ${ }^{3}$ Department of Biology and Genetics, Medical University of Gdansk, Poland \\ ${ }^{4}$ Laboratory of Clinical Genetics, University Clinical Center, Gdansk, Poland
}

This paper was guest edited by Prof. Krzysztof J. Filipiak

\begin{abstract}
Background: Severe familial hypercholesterolemia $(F H)$ individuals, refractory to conventional lipidlowering medications are at exceptionally high risk of cardiovascular events. The established therapeutic option of last choice is lipoprotein apheresis (LA). Herein, it was sought to investigate the clinical usefulness of LA in a highly selected group of severe heterozygous $\mathrm{FH}(\mathrm{HeFH})$, as recently described by the International Atherosclerosis Society (IAS), for their efficacy in lipid reduction and safety.

Methods: Efficacy and safety of LA were investigated in 318 sessions of 7 severe HeFH females with cardiovascular disease, over a mean period of $26.9 \pm 6.5$ months. Relative reduction of low density lipoprotein cholesterol $(L D L-C) \geq 60 \%$, clinical complications and vascular access problems were evaluated and compared between the direct adsorption of lipoproteins (DALI) and lipoprotein filtration (Membrane Filtration Optimized Novel Extracorporeal Treatment [MONET]). Additionally, lipoprotein (a) [Lp(a)], total cholesterol (TC), high density lipoprotein cholesterol (HDL-C), triglycerides (TG) and fibrinogen concentrations were investigated.

Results: The relative reduction of $L D L-C, T C, T G$ and $L p(a)$ were $69.4 \pm 12.9 \%, 59.7 \pm 9.1,51.5 \pm$ $\pm 14.2 \%$ and $71.3 \pm 14.4 \%$, respectively. A similar efficacy was found in both systems in LDL-C removal. DALI system led to larger depletions of $L p(a)(80.0[76-83] \%$ vs. $73.0[64.7-78.8] \% ; p<0.001)$. The frequency of clinical side effects and vascular access problems were low (8.5\%).

Conclusions: Long-term LA in severe HeFH individuals is safe and efficiently reduces LDL-C and $L p(a)$. Higher efficacy of the DALI system than MONET in Lp (a) removal may indicate the need for individualized application of the LA system in severe HeFH individuals. (Cardiol J 2019; 26, 6: 669-679) Key words: lipoprotein apheresis, severe familial hypercholesterolemia, lipoprotein (a)
\end{abstract}

Address for correspondence: Agnieszka Mickiewicz, MD, PhD, $1^{\text {st }}$ Department of Cardiology, Medical University of Gdansk, ul. Dębinki 7, 80-952 Gdańsk, Poland, tel: +48 5834925 04, e-mail: agnieszka.mickiewicz@gumed.edu.pl 


\section{Introduction}

Severe familial hypercholesterolemia $(\mathrm{FH})$ patients are at exceptionally high risk of cardiovascular disease (CVD) [1]. Although some authors have suggested that individuals with heterozygous $\mathrm{FH}(\mathrm{HeFH})$ and untreated low-density lipoprotein cholesterol (LDL-C) greater than 309 $\mathrm{mg} / \mathrm{dL}$ suffer from severe $\mathrm{HeFH}$, the definition of severe phenotype of $\mathrm{FH}$ was not clear [2]. Recently, the International Atherosclerosis Society (IAS) considered not only initial LDL-C values, but also the presence of risk conditions, as well as clinical or advanced subclinical atherosclerotic CVD [2-7]. These high-risk conditions proposed by IAS include diabetes, arterial hypertension, smoking history, chronic kidney disease, positive family history of early CVD in first-degree relative, low high-density lipoprotein cholesterol (HDL-C) and elevated lipoprotein (a) [Lp(a)]. Among high risk features special attention should be directed to increased $\mathrm{Lp}(\mathrm{a})$ with its strong atherogenic and thrombogenic effect, and resistance to conventional lipid-lowering medications [5, 8]. Individuals with severe phenotype of $\mathrm{FH}$ often do not adequately respond to high-intensity lipid-lowering medications and do not achieve treatment goals [9]. The recommended target level for severe $\mathrm{FH}$ individuals for secondary prevention is LDL-C below $70 \mathrm{mg} / \mathrm{dL}$ [9]. Thus, more aggressive forms of therapy might be beneficial in severe $\mathrm{FH}$ to arrest the progression of atherosclerosis and reduce cardiovascular event rate [10-12]. The treatment option in severe $\mathrm{FH}$ widely used since the 80 s has been long-term lipoprotein apheresis (LA) [13, 14]. LA is an extracorporeal technique of selective removal of lipoproteins. Various lipoprotein apheresis systems are routine in clinical use currently. Whole blood adsorption of lipoproteins includes direct adsorption of lipoproteins with polyacrylamide (DALI) and dextran sulfate cellulose adsorption. Atherogenic lipoproteins may also be eliminated in following primary plasma separation methods: lipoprotein filtration (MONET), heparin-induced extracorporeal LDL-C precipitation (HELP), silicate gel adsorption, immunoadsorption (IMA) and dextran sulfate cellulose [15]. LA procedures need to be repeated every $1-2$ weeks due to LDL-C and $L p(a)$ level rebound effect. All LA techniques have been shown to effectively reduce LDL-C along with $\mathrm{Lp}(\mathrm{a})$ concentrations by more than $60 \%$, while being well tolerated in long-term application. LDL-apheresis treatment also exerts a pleiotropic effect, improving rheological properties of the blood and reducing inflammatory markers [16, 17]. Regular apheresis sessions have been proved to slow the progression of atherosclerosis and reduce the incidence of cardiovascular events [12, 18-20]. The main goal of LA treatment in HeFH is to achieve an LDL-C reduction $\geq 60 \%$ at each therapeutic session [21]. Additionally, time-averaged LDL-C less than $100 \mathrm{mg} / \mathrm{dL}$ might be considered as a goal [21].

Although data confirming the effectiveness and safety of lipoprotein apheresis have been published, the studies often aggregated patients with undefined hypercholesterolemia, homozygous $\mathrm{FH}, \mathrm{HeFH}$ and isolated increased $\mathrm{Lp}(\mathrm{a})$, treated with various apheresis systems and differing lipidlowering medication regimens [22, 23]. This lack of stratification by type of dyslipidemia resulted in misleading findings. Therefore, the main purpose of the current research was to investigate the clinical usefulness of lipoprotein apheresis in a highly selected group of severe $\mathrm{HeFH}$, according to the IAS definition, for their efficacy in lipid reduction and safety. Additionally, the safety and effectiveness of DALI and MONET systems were compared in the current study group.

\section{Methods}

The study was carried out prospectively in a large Polish lipoprotein apheresis center, established at the First Department of Cardiology, Medical University of Gdansk, following the Good Clinical Practice guidelines. Researchers obtained written informed consent before patient inclusion, in accordance with the Declaration of Helsinki. All treatment protocols and medical records for each patient undergoing LA were reviewed, except for initial sessions in the first month of treatment.

\section{Patients}

The primary indication to LA treatment was $\mathrm{HeFH}$ with symptomatic CVD and LDL-C concentration of more than $160 \mathrm{mg} / \mathrm{dL}$ despite maximally tolerated intensive lipid-lowering medications [14]. Seven female patients with definite $\mathrm{FH}$ according to the modified Dutch Lipid Network Criteria and confirmed a mutation in $L D L R$ or $A P O B$ gene, fulfilling the criteria of severe $\mathrm{FH}$ by the IAS, were enrolled into the study [4, 24]. At the initiation of LA treatment, all patients had a history of a documented CVD and at least three additional high risk-features for severe $\mathrm{FH}$. All individuals were treated with rosuvastatin in a dose of $40 \mathrm{mg}$ daily with or without ezetimibe $10 \mathrm{mg}$ daily by more than 12 months before starting LA and continued such 
treatment on apheresis. The mean age of patients at the start of LA therapy was $54.5 \pm 5.5$ years. Detailed clinical and biochemical characteristics of investigated patients are presented in Table 1.

\section{Lipoprotein apheresis}

Lipoprotein apheresis sessions $(n=318)$ were performed in weekly or biweekly intervals using two techniques, according to patient characteristics over a period of 37 months. Concomitant angiotensin converting enzyme inhibitors (ACEI) therapy was a contraindication to DALI treatment. LA therapy was started $6.0 \pm 1.5$ years after the clinical diagnosis of FH. 162 DALI sessions with large adsorber configurations (DALI 1000, DALI 1250) and 156 MONET procedures were performed over a mean period of $26.9 \pm 6.5$ months [25]. To provide adequate effectiveness, at least 1.5 of blood volume was processed during DALI therapeutic sessions, and at least $45 \mathrm{~mL}$ of plasma volume $/ \mathrm{kg}$ was treated during MONET sessions [26]. Both acid citrate dextrose (ACD-A) and heparin in the priming solution were used as an anticoagulant. Arteriovenous (AV) fistula was established as access in all patients due to the insufficiency of peripheral venous access. Initial sessions in the first months and procedures interrupted before expected blood/ /plasma volume purification were excluded from the final analysis of biochemical parameters.

\section{Biochemical parameters}

All biochemical parameters were measured in one laboratory at scheduled intervals. LDL-C levels were subsequently calculated using the Friedewald formula unless triglycerides (TG) were above $400 \mathrm{mg} / \mathrm{dL}$. Acute reduction in total cholesterol (TC), LDL-C, HDL-C, TG, Lp(a) and fibrinogen were calculated from pre- and post-apheresis results. The time-averaged mean LDL-C level was calculated according to the formula devised by Kroon, where $C_{\mathrm{MAX}}$ and $C_{\mathrm{MIN}}$ are defined as the immediate pre- and post-treatment values: $C_{\mathrm{AVG}}=$ $C_{\mathrm{MIN}}+0.73 \times C_{\mathrm{MAX}}-C_{\mathrm{MIN}}$ [27]. The effectiveness of LA was expressed as an achievement of acute post-apheresis LDL-C reduction by more than $60 \%$. Alternatively, the time-averaged LDL-C below $100 \mathrm{mg} / \mathrm{dL}$ was a goal of treatment. Additionally, HDL-C, TC, TG, Lp(a) and fibrinogen reductions were investigated.

\section{Side-effects}

Clinical complications and vascular access problems were investigated at each therapeutic apheresis session. Clinical complications were

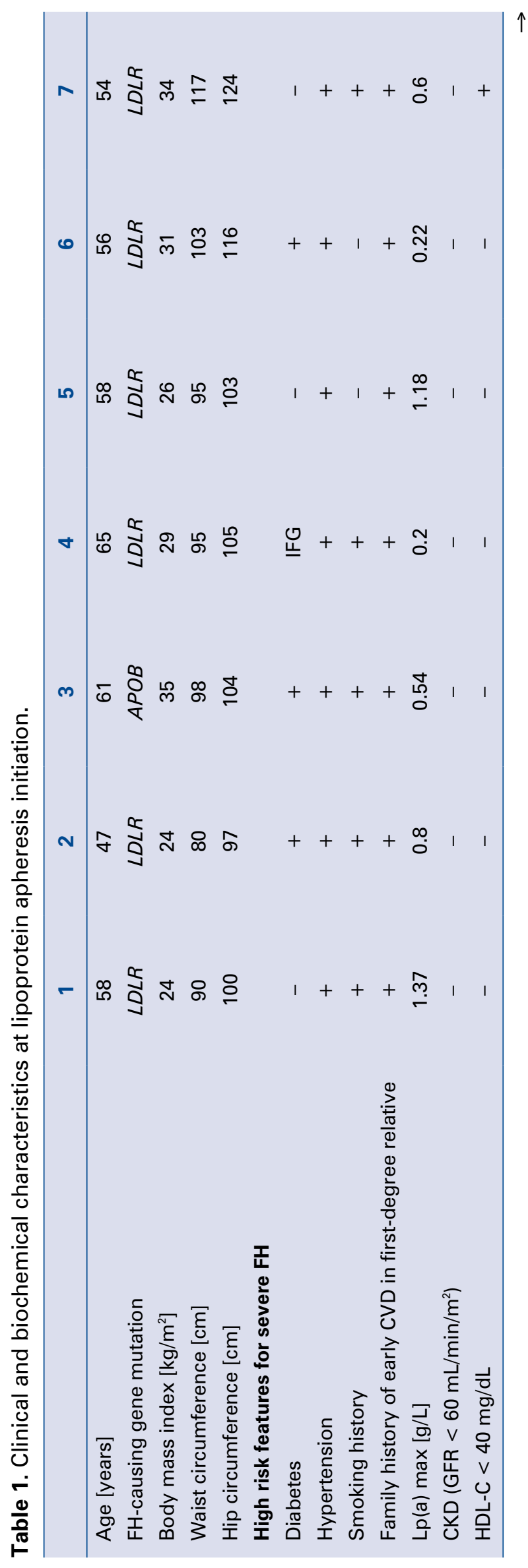


Cardiology Journal 2019, Vol. 26, No. 6

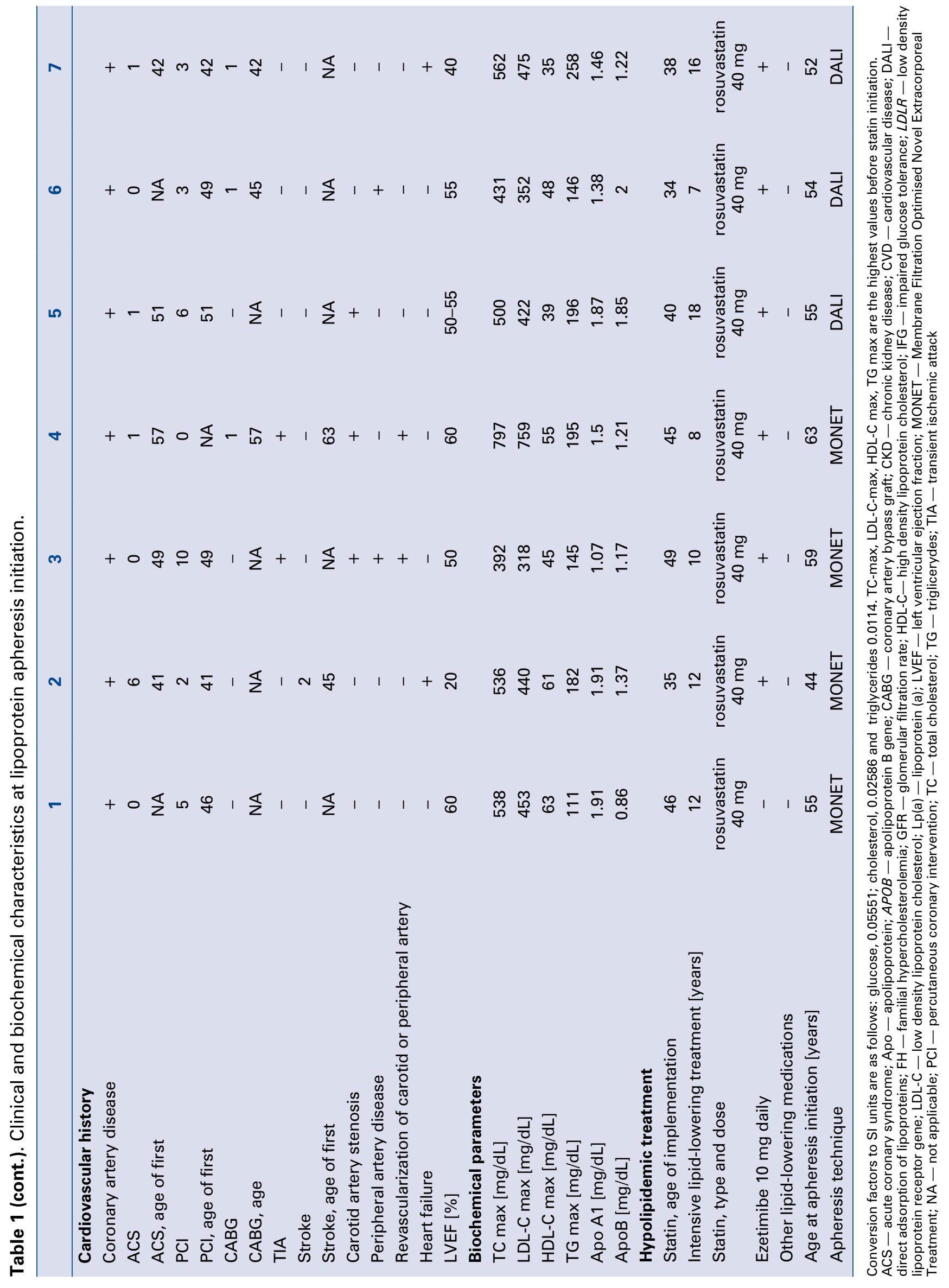


Table 2. Lipoprotein apheresis — biochemical parameters.

\begin{tabular}{lcccc}
\hline & Mean \pm SD & Median & Minimum & Maximum \\
\hline TC pre-apheresis [mg/dL] & $308.9 \pm 94.1$ & 294.0 & 153.0 & 569.0 \\
TC post-apheresis [mg/dL] & $121.6 \pm 39.3$ & 114.0 & 62.0 & 354.0 \\
TC [\% reduction] & $59.7 \pm 9.1$ & 61.5 & 32.4 & 78.9 \\
LDL-C pre-apheresis [mg/dL] & $222.8 \pm 89.5$ & 207.5 & 54.0 & 490.0 \\
LDL-C post-apheresis [mg/dL] & $68.8 \pm 37.6$ & 66.0 & 1.0 & 177.0 \\
LDL-C [\% reduction] & $69.4 \pm 12.9$ & 71.8 & 17.3 & 98.3 \\
Interval LDL-C [mg/dL] & $181.4 \pm 72.8$ & 154.9 & 40.0 & 395.8 \\
HDL-C pre-apheresis [mg/dL] & $42.5 \pm 10.1$ & 41.0 & 15.0 & 75.0 \\
HDL-C post-apheresis [mg/dL] & $32.4 \pm 6.8$ & 32.0 & 11.0 & 53.0 \\
HDL-C [\% reduction] & $22.7 \pm 10.9$ & 21.7 & 0.0 & 62.16 \\
TG pre-apheresis [mg/dL] & $220.8 \pm 162.7$ & 157.0 & 46.0 & 1121.0 \\
TG post-apheresis [mg/dL] & $103.8 \pm 84.8$ & 72.0 & 23.0 & 616.0 \\
TG [\% reduction] & $51.5 \pm 14.2$ & 53.2 & 10.8 & 83.6 \\
Lp(a) pre-apheresis [g/L] & $0.5 \pm 0.4$ & 0.39 & 0.08 & 1.37 \\
Lp(a) post-apheresis [g/L] & $0.12 \pm 0.09$ & 0.09 & 0.01 & 0.57 \\
Lp(a) [\% reduction] & $71.3 \pm 14.4$ & 76.1 & 21.4 & 94.1 \\
Fibrinogen pre-apheresis [mg/dL] & $3.25 \pm 0.63$ & 3.25 & 2.2 & 6.47 \\
Fibrinogen post-apheresis [mg/dL] & $1.96 \pm 0.77$ & 2.02 & 0.63 & 3.96 \\
Fibrinogen [\% reduction] & $39.7 \pm 21.2$ & 45.6 & 2.8 & 76.0 \\
\hline
\end{tabular}

Data are presented as mean \pm standard deviation (SD) and median with minimum and maximum. Abbreviations - see Table 1.

specified as follows: hypotension with systolic blood pressure $<90 \mathrm{mmHg}$ and accompanying symptoms (paleness, nausea), hypocalcemia, oedema, severe bleeding, anemia, and thrombocytopenia. Vascular complications included puncture problems, hematoma, bleeding and stenosis of AV fistula.

\section{Statistical analysis}

Continuous data were presented as a mean value and standard deviation (SD) or as a median and interquartile range (IQR) or as a median and minimum and maximum value. Categorical data were presented as percentages. Normal distribution was verified by the Kolmogorov-Smirnov test. Continuous data were compared by the Student t-test or U-Mann Whitney test depending on the distribution. Categorical data were compared by the $\chi^{2}$ test and Fisher exact test. P value less than 0.05 was considered statistically significant. Data were analyzed using SPSS software v.21 (IBM, Chicago, Illinois, USA).

\section{Results}

\section{Lipoprotein apheresis}

The mean duration time of the procedure was $140.0(60-240) \mathrm{min}$. ACD-A was used in a mean volume of $329.8 \pm 134.9 \mathrm{~mL}$, with the ratio of citrate: blood ranging from 1:20 to 1:40 in both DALI and MONET. DALI sessions were significantly shorter than MONET (130.0 [120-140] vs. 170.0 [158.0-183.7]; $\mathrm{p}<0.001)$ with less ACD-A consumption (244.0 [215-302] vs. 431.0 (374.5-486.5); $\mathrm{p}<0.001)$. Average blood volume processed during DALI sessions was $8540 \pm 155 \mathrm{~mL}$. During MONET sessions average plasma volume $2903.5 \pm 867.1 \mathrm{~mL}$ was achieved.

\section{Cholesterol, lipoprotein (a) and fibrinogen}

Laboratory parameters before and during chronic LA treatments are summarized in Table 2. Mean pre-apheresis values of TC and LDL-C were high $(308.9 \pm 94.1$ and $222.8 \pm 89.5 \mathrm{mg} / \mathrm{dL}$, respectively). Apheresis reduced both lipids acutely to $121.6 \pm 39.3 \mathrm{mg} / \mathrm{dL}$ and $68.8 \pm 37.6$ $\mathrm{mg} / \mathrm{dL}$, respectively. Mean pre-apheresis values of HDL-C were below the normal range for females $(42.5 \pm 10.1 \mathrm{mg} / \mathrm{dL})$. Apheresis reduced HDL-C to a lesser extent than other lipids $(22.7 \pm 10.9 \%)$. Apheresis sessions removed TG by $51.5 \pm 14.2 \%$ in the mean, starting from $220.8 \pm 162.7 \mathrm{mg} / \mathrm{dL}$. Apheresis session led to acute depletion of $\mathrm{Lp}(\mathrm{a})$ from pre-apheresis concentration of $0.5 \pm 0.4 \mathrm{~g} / \mathrm{L}$ to $0.12 \pm 0.09 \mathrm{~g} / \mathrm{L}$. Fibrinogen was reduced by 
Table 3. Biochemical parameters in DALI versus MONET system.

\begin{tabular}{lccc}
\hline & DALI & MONET & P \\
\hline TC pre-apheresis [mg/dL] & $238.0(207-295)$ & $372.0(254.5-409.5)$ & $<0.001$ \\
TC post-apheresis [mg/dL] & $100.0(83-121)$ & $114.0(101.5-134.5)$ & 0.003 \\
TC [\% reduction] & $58.8(54.8-64.5)$ & $65.6(61.2-69.7)$ & $<0.001$ \\
LDL-C pre-apheresis [mg/dL] & $141.0(122-170)$ & $286.0(191.5-326.5)$ & $<0.001$ \\
LDL-C post-apheresis [mg/dL] & $37.5(26-57)$ & $69.0(49.5-88.0)$ & $<0.001$ \\
LDL-C [\% reduction] & $72.6(66.5-83.5)$ & $74.1(69.9-77.9)$ & 0.8 \\
Interval LDL-C [mg/dL] & $113.4(96.9-137.5)$ & $228.5(155.6-260.3)$ & $<0.001$ \\
HDL-C pre-apheresis [mg/dL] & $36.0(34-39)$ & $50.0(42.5-58.0)$ & $<0.001$ \\
HDL-C post-apheresis [mg/dL] & $31.0(28.2-34.0)$ & $36.0(30.0-40.0)$ & $<0.001$ \\
HDL-C [\% reduction] & $14.3(9.5-20.6)$ & $30.3(25.7-34.2)$ & $<0.001$ \\
TG pre-apheresis [mg/dL] & $285.0(184-383)$ & $132.0(104.0-185.0)$ & $<0.001$ \\
TG post-apheresis [mg/dL] & $126.50(73-198)$ & $63.0(49.5-81.5)$ & $<0.001$ \\
TG [\% reduction] & $51.81(45.2-62.2)$ & $54.4(44.3-62.6)$ & 0.9 \\
Lp(a) pre-apheresis [g/L] & $0.43(0.22-0.72)$ & $0.77(0.35-1.09)$ & 0.001 \\
Lp(a) post-apheresis [g/L] & $0.08(0.04-0.13)$ & $0.17(0.12-0.24)$ & $<0.001$ \\
Lp(a) [\% reduction] & $80.0(76-83)$ & $73.0(64.7-78.8)$ & $<0.001$ \\
\hline
\end{tabular}

Data are presented as median (interquartile range [IQR]). Abbreviations - see Table 1.

$39.7 \pm 21.2 \%$ starting from the pre-apheresis level of $3.25 \pm 0.63 \mathrm{mg} / \mathrm{dL}$ (Table 2 ).

\section{DALI vs. MONET}

Higher pre- and post-apheresis TC, LDL-C, HDL-C and $\mathrm{Lp}(\mathrm{a})$ concentrations in MONET sessions vs. DALI (Table 3 ) were observed. In contrast, the pre- and post-apheresis TG levels were higher in patients treated by DALI system, compared to MONET (Table 3). MONET system led to higher TC and HDL-C reductions, compared to DALI (Fig. 1).

The pre-apheresis concentrations of fibrinogen were similar in DALI and MONET groups. In comparison to MONET, DALI treatment led to a lower removal of fibrinogen (62.5 [52.1-68.0]\% vs. 19.8 [13.9-25.2]\%; $\mathrm{p}<0.001$ ) (Fig. 1). Post-apheresis fibrinogen concentration in MONET sessions was below normal range $(1.15$ [1.00-1.52] mg/dL).

Analyzing all LA therapeutic sessions, the relative reduction of LDL-C up to $69.4 \pm 12.9 \%$ (71.8 [17.3-98.3]\%) was achieved. Comparing both systems, their similar efficacy was found $(72.6$ [66.5-83.5]\% vs. 74.1 [69.9-77.9]\%; $\mathrm{p}=0.809$ ) (Fig. 2). A large number of LA sessions resulted in at least a $60 \%$ reduction of LDL-C ( $82 \%$ of DALI treatments and $78 \%$ of MONET treatments).

Calculated time-averaged LDL-C was $181.4 \pm$ $\pm 72.8(154.9$ [40-395.8]) $\mathrm{mg} / \mathrm{dL}$. The DALI system resulted in the achievement of a lower time-averaged LDL-C, than MONET (113.4 vs. $228.5 \mathrm{mg} / \mathrm{dL} ; \mathrm{p}<0.001$ ).

The acute reduction of $L p(a)$ of $71.3 \pm 14.4 \%$ (76.1 [21.4-94.1]\%) was achieved. It was observed that DALI system was more efficient in relative removal of $\mathrm{Lp}(\mathrm{a})$ than MONET $(80.0$ [76-83]\% vs. 73.0 [64.7-78.8]\%; $<$ 0.001) (Fig. 2).

Additional analysis revealed that $54 \%$ of all sessions resulted in a post-apheresis LDL-C of less than $70 \mathrm{mg} / \mathrm{dL}$. A higher percent of DALI vs. MONET sessions resulted in decreasing LDL-C below $70 \mathrm{mg} / \mathrm{dL}$ ( $88 \%$ vs. $32 \%$ ).

\section{Side effects}

The total incidence of clinical side effects was low $(8.5 \%)$. Major complications were observed in 2 cases of DALI treatment. One episode of bradykinin syndrome (hypotension, flush, bradycardia and dyspnea) with Quincke odema and lumbar pain was observed. The patient was switched to MONET system. Heparin-induced thrombocytopenia (HIT) with thrombosis in extracorporeal system appeared in another individual. Heparin was replaced by fondaparinux and DALI treatment was continued.

The total incidence of hypotension, vascular problems, and hypocalcemia was low $(7.5 \%, 6.12 \%$, $1.7 \%$, respectively). However, the frequency of complications related to vascular access was higher in MONET sessions vs. DALI (10.5\% vs. $1.4 \%$, 


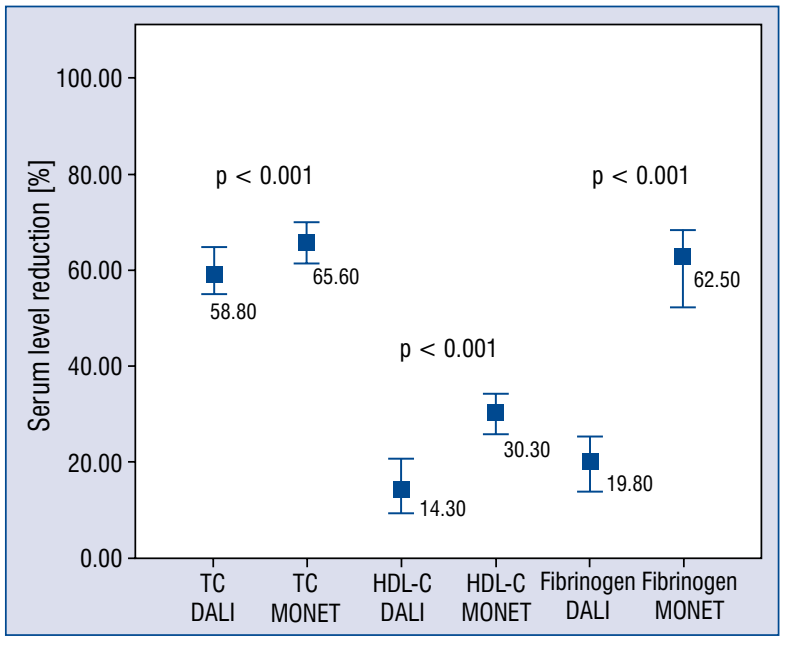

Figure 1. Reduction of total cholesterol (TC), high density lipoprotein cholesterol (HDL-C) and fibrinogen in DALI vs. MONET. Data are presented as median and interquartile range.

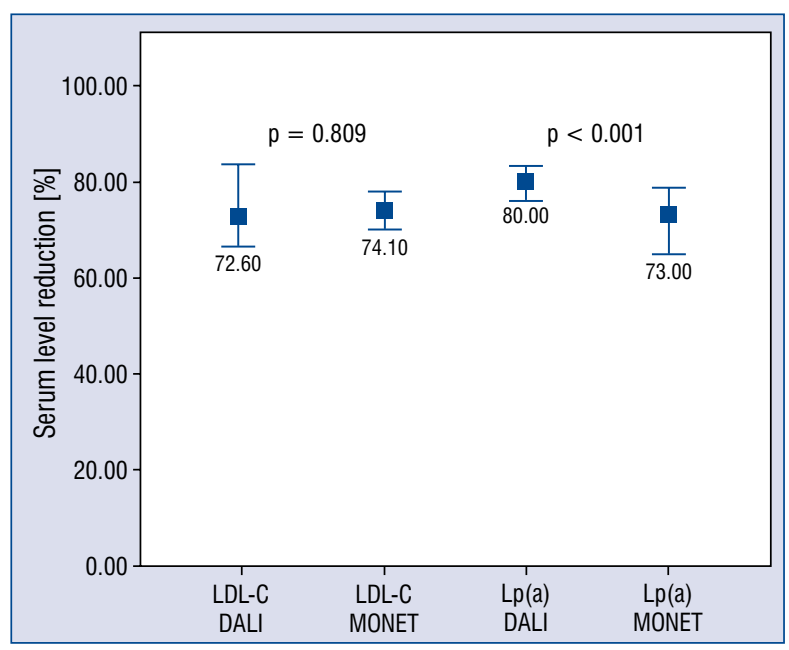

Figure 2. Reduction of low density lipoprotein cholesterol (LDL-C) and lipoprotein (a) [Lp(a)] in DALI vs. MONET. Data are presented as a median and interquartile range. $\mathrm{p}=0.001)$. Mild, transient hypotension occurred with similar frequency in DALI and MONET (4.9\% vs. $9.9 \%, p=0.12$ ). Administration of crystalloid infusion before and during apheresis substantially reduced the frequency of symptomatic hypotension. Blood flow at the start and the end of procedures was not related with the incidence of hypotension (Table 4). Hypocalcemia incidence rate was similar during MONET sessions (3.3\% vs. $0 \%, \mathrm{p}=0.06$ ). Anemia occurred in one individual undergoing DALI and one treated by MONET system. Angina episodes were not recorded, as well as abdominal pain.

\section{Discussion}

The study was undertaken to assess the efficacy and safety of lipoprotein apheresis in a highly selected group of severe $\mathrm{FH}$ individuals, by IAS definition. Investigated patients presented a pattern of severe $\mathrm{HeFH}$ with an advanced CVD, high LDL-C at initial presentation ( $>310 \mathrm{mg} / \mathrm{dL}$ ) and at least three additional high-risk features. Increased $\mathrm{Lp}$ (a) level greater than $50 \mathrm{mg} / \mathrm{dL}$ was present in more than half of them (4/7) [28]. Administration of high-intensity oral lipid-lowering agents failed to reduce LDL-C below $160 \mathrm{mg} / \mathrm{dL}$. Although cardiovascular risk in $\mathrm{HeFH}$ is largely driven by chronic exposure to elevated LDL-C, cardiovascular risk factors in $\mathrm{HeFH}$ are additive, indicating very high CVD risk in the present cohort. Therefore, treatment strategy should be aggressive, targeting an ideal goal of LDL-C below $70 \mathrm{mg} / \mathrm{dL}$. In the current study, LDL-C was acutely reduced from $223 \mathrm{mg} /$ $/ \mathrm{dL}$ to $69 \mathrm{mg} / \mathrm{dL}$, which corresponded to a relative reduction of $69 \%$. Relative decreases of LDL-C greater than $60 \%$ was reached in a large number of treatments (close to 80\%) indicating a good quality of treatment. In LDL-Apheresis Atherosclerosis Regression Study (LAARS) the achieved acute $63 \%$ reduction in LDL-C led to the angiographic

Table 4. Influence of crystalloid infusion on hypotension during lipoprotein apheresis (LA) treatment.

\begin{tabular}{lccc}
\hline & $\begin{array}{c}\text { Symptomatic hypotension } \\
\text { during apheresis (n= 22) }\end{array}$ & $\begin{array}{c}\text { Apheresis session without } \\
\text { hypotension (n = 272) }\end{array}$ & P \\
\hline Crystalloid infusion & $14(63.63 \%)$ & $54(19.85 \%)$ & $<0.001$ \\
Crystalloid infusion before procedure & $8(36.36 \%)$ & $39(14.34 \%)$ & 0.01 \\
Crystalloid infusion during procedure & $9(40.91 \%)$ & $20(7.35 \%)$ & $<0.001$ \\
Blood flow at start of LA [mL/min] & $52.86 \pm 9.16$ & $50.81 \pm 8.71$ & 0.3 \\
Blood flow 2 at the end of LA [mL/min] & $96.00 \pm 29.00$ & $101.17 \pm 20.25$ & 0.4 \\
\hline
\end{tabular}

Data for blood flow are presented as mean \pm standard deviation. 
arrest of the progression of coronary artery disease in a majority of patients treated with simvastatin and biweekly LA [29]. Other studies carried out in $\mathrm{HeFH}$, on various systems of apheresis reported $55-70 \%$ mean LDL-C reduction [21, 30, 31]. Nevertheless, it was found that post-apheresis LDL-C values were below $70 \mathrm{mg} / \mathrm{dL}$ only in $54 \%$ of LA sessions. When DALI and MONET systems were compared, single DALI sessions achieved LDL below $70 \mathrm{mg} / \mathrm{dL}$ more often when compared to MONET ( $88 \%$ vs. $32 \%$ ). These results may be easily explained by higher pre-apheresis LDL-C observed in patients treated with MONET as compared to DALI. Premature discontinuation of MONET sessions due to adverse events decreased the percent of efficacious LA sessions, compared to other reports [31]. Last but not least, the staff and site experience are a known factor influencing the course of LA sessions [32].

Considering the achievement of a time-averaged LDL-C below $100 \mathrm{mg} / \mathrm{dL}$ as a goal of LA therapy, it was out of range in the present study $(181 \mathrm{mg} / \mathrm{dL})$. However, it agrees with previous studies. In a large study of 118 patients treated by LA in Dresden, the time-averaged LDL-C was $119.8 \mathrm{mg} / \mathrm{dL}$, similar to the present DALI patients. It may be explained by lower pre-apheresis LDL-C in the Dresden group, than the cohort herein (148.8 mg/dL vs. $223 \mathrm{mg} / \mathrm{dL}$ ) [33].

The mean reduction of $\mathrm{Lp}$ (a) observed in this study was close to $70 \%$. DALI treatment led to larger depletion of $\mathrm{Lp}(\mathrm{a})$ than MONET. Ramlow et al. [31] showed equal $\mathrm{Lp}(\mathrm{a})$ removal and slightly better efficacy of LDL-C removal for DALI treatment than MONET. It is suspected that a more efficient removal of $\mathrm{Lp}$ (a) in the present DALI system might be an effect of an application of larger adsorbers (DALI 1000 and 1250) or higher blood volume processed. However, there are other factors determining the acute lipoprotein reduction in DALI-apheresis such as weight, height, preapheresis lipid levels, as well as blood flow rate through the adsorber.

The present findings also confirmed that direct adsorption and lipoprotein filtration varied in selectivity. MONET substantially reduced fibrinogen concentration, which may improve blood viscosity and its rheological properties. Bleeding complications were not reported, even though $2 \mathrm{pa}$ tients were administrated with oral anticoagulants. MONET system led to slightly higher HDL-C reduction compared to DALI. However, the reduction was lower, than other lipids. Thus, results agree with previous reports [31].
A mean rate of side effects of $8.5 \%$ was observed, which is in the line with data from a large study by Dittrich-Riediger et al. [33]. Serious AE were incidental as in previous reports [34]. Despite ACEI cessation before DALI initiation, bradykinin syndrome was reported in the present study. Another patient treated by DALI was affected by HIT. DALI system is known to cause bradykinin release with peaks at $1000-2000 \mathrm{~mL}$ of treated blood volume and ACEI block bradykinin degradation into inactive metabolites. Thus, they are contraindicated in patients treated by DALI system. Angiotensin receptor blockers may be administrated, as in the present case. HIT is an extremely rare complication of LA. However, some authors reported thrombocytopenia previously. The most frequently observed complications of lipoprotein apheresis in the present study were vascular access problems and hypotension, as previously reported by other authors $[33,35]$. A higher incidence of vascular access problems was found compared to other studies [23]. In a large multicenter, prospective study of German patients undergoing DALI and MONET apheresis, KozikJaromin et al. [35] reported 27 puncture problems in 3451 sessions [35]. However, hematoma and bleeding as problems with vascular access were also reported herein. Otherwise, some data indicate an increased rate of venous puncture problems were found in female vs. male patients [33]. All investigated individuals in the present cohort were females. Secondly, the type of the vascular access determined issues with its maintenance. Accessing peripheral veins might be the best option for lipoprotein apheresis treatment [36]. In the United Kingdom analysis of peripheral vein cannulation represented even $79 \%$ of initial vascular access strategies with $\mathrm{AV}$ fistula use accounting for $15 \%$, with a trend to AV cannulation [37]. Unfortunately, due to unavailability of large veins for repeated puncture, arteriovenous fistulas were established in all patients of the present study. Detailed analysis showed that 1 patient undergoing MONET suffered from recurrent stenosis and thrombosis of arteriovenous fistula. Kozik-Jaromin et al. [35] excluded the first 3 months of treatment from analysis, not only the first month of treatment as in the present study. Increased rate of AE during all 12 months of treatment was observed in previous reports.

Mild, transient hypotension, mainly caused by initial "blood donation" into the extracorporeal circuit, occurred with $7.5 \%$ frequency. The German Registry of Lipoprotein Apheresis (GLAR) showed a lower rate of hypotension of $1.09-1.28 \%$ [21]. However, as demonstrated herein, hypoten- 
sion may be avoided by crystalloid infusion to the contralateral vein before and during an apheresis session. Some authors have described routine intravenous administration of saline or HAES at apheresis session initiation, and this may have been the reason for this difference. Symptomatic hypocalcemia caused by citrate infusion was rare in the present study. Oral supplementation of calcium prior to a DALI session was introduced in susceptible patients, as well as routine optimization of ACD-A.

Long-term LA with efficient LDL-C and Lp(a) removal was a consequence to improvement of the CVD course in the patients studied. The incidence of major adverse cardiac events (MACE; defined as cardiovascular death, non-fatal acute coronary syndrome and repeat coronary revascularization) decreased after LA initiation. MACE rate was reduced from 41 events before LA inception to 8 during the period of LA treatment. The positive impact of LDL apheresis on cardiovascular morbidity in individuals with hypercholesterolemia has been previously confirmed in several observational trials [38]. Sampietro et al. [39] reported a significant reduction of adverse cardiac or vascular events in 30 individuals with $\mathrm{FH}$ or familial combined hypercholesterolemia and CVD. Adverse cardiac or vascular events incidences occurred prior and after LA treatment inception, which were 86 and 15 events, respectively [39].

According to available research, this is the first report focused on the most severe phenotype of $\mathrm{HeFH}$ refractory to an equal regimen of statin (rosuvastatin $40 \mathrm{mg}$ daily) at particularly high cardiovascular risk. All patients were female. Thus, gender influence on cardiovascular risk can be omitted. LA in the present study was carried out and documented by one physician at a specialized apheresis center. The treatment and observation period were long.

Study results highlight the importance of more aggressive forms of treatment such as LA in severe $\mathrm{HeFH}$ individuals with advanced CVD, additional high-risk features, and LDL-C greater than 160 $\mathrm{mg} / \mathrm{dL}$, despite high-intensity statin therapy. The present findings also point to the fact that despite LA there was a substantial unmet need for novel schedules of treatment to control LDL-C in those individuals [4]. In further studies on cardiovascular outcome in real-world practice, it might be interesting to clarify if severe $\mathrm{HeFH}$ individuals with $\mathrm{CVD}$ and increased $\mathrm{Lp}$ (a) concentration benefit from DALI treatment with large adsorbers (1000 or 1250) in combination with novel drugs [40].

\section{Limitations of the study}

The present study is small in size, which was caused by a low number of $\mathrm{HeFH}$ patients treated by lipoprotein apheresis in Poland. Despite an increase in the proportion of patients treated with strong statins in recent years, treatment goals in hypercholesterolemia are not being achieved [41]. Based on the prevalence of severe FH eligible to LA of $2.4 \%$ and approximately $1000 \mathrm{FH}$ individuals with molecular confirmation in Poland (unpublished data), it is estimated that there are 24 severe $\mathrm{HeFH}$, of which 7 are currently being treated with LA [42].

\section{Conclusions}

Long-term LA in severe $\mathrm{HeFH}$ individuals is safe and efficiently reduces LDL-C and $\mathrm{Lp}(\mathrm{a})$. Higher efficacy of DALI system vs. MONET in $\mathrm{Lp}$ (a) removal may indicate a need for individualized application of LA system in severe $\mathrm{HeFH}$ individuals.

\section{Acknowledgements}

This research did not receive any grants from funding agencies in public, commercial, or notfor-profit sectors. We thank all the nurses who participated in lipoprotein apheresis treatment.

\section{Conflict of interest: None declared}

\section{References}

1. Neil A, Cooper J, Betteridge J, et al. Reductions in all-cause, cancer, and coronary mortality in statin-treated patients with heterozygous familial hypercholesterolaemia: a prospective registry study. Eur Heart J. 2008; 29(21): 2625-2633, doi: 10.1093/ eurheartj/ehn422, indexed in Pubmed:18840879.

2. Besseling J, Kindt I, Hof M, et al. Severe heterozygous familial hypercholesterolemia and risk for cardiovascular disease: a study of a cohort of 14,000 mutation carriers. Atherosclerosis. 2014; 233(1): 219-223, doi: 10.1016/j.atherosclerosis.2013.12.020, indexed in Pubmed: 24529147.

3. Tada H, Kawashiri Ma, Okada H, et al. Assessment of coronary atherosclerosis in patients with familial hypercholesterolemia by coronary computed tomography angiography. Am J Cardiol. 2015; 115(6): 724-729, doi: 10.1016/j.amjcard.2014.12.034, indexed in Pubmed: 25618577.

4. Santos R, Gidding S, Hegele R, et al. Defining severe familial hypercholesterolaemia and the implications for clinical management: a consensus statement from the International Atherosclerosis Society Severe Familial Hypercholesterolemia Panel. Lancet Diabetes Endocrinol. 2016; 4(10): 850-861, doi: 10.1016/ s2213-8587(16)30041-9.

5. Alonso R, Andres E, Mata N, et al. Lipoprotein(a) levels in familial hypercholesterolemia: an important predictor of cardiovascular disease independent of the type of LDL receptor muta- 
tion. J Am Coll Cardiol. 2014; 63(19): 1982-1989, doi: 10.1016/j. jacc.2014.01.063, indexed in Pubmed: 24632281.

6. Jansen ACM, van Aalst-Cohen ES, Tanck MW, et al. The contribution of classical risk factors to cardiovascular disease in familial hypercholesterolaemia: data in 2400 patients. J Intern Med. 2004; 256(6): 482-490, doi: 10.1111/j.1365-2796.2004.01405.x, indexed in Pubmed: 15554949.

7. Chan DC, Pang J, Hooper AJ, et al. Elevated lipoprotein(a), hypertension and renal insufficiency as predictors of coronary artery disease in patients with genetically confirmed heterozygous familial hypercholesterolemia. Int J Cardiol. 2015; 201: 633-638, doi: 10.1016/j.ijcard.2015.08.146, indexed in Pubmed: 26340131.

8. Nordestgaard BG, Chapman MJ, Ray K, et al. Lipoprotein(a) as a cardiovascular risk factor: current status. Eur Heart J. 2010; 31(23): 2844-2853, doi:10.1093/eurhearti/ehq386, indexed in Pubmed: 20965889.

9. Perez de Isla L, Alonso R, Watts GF, et al. Attainment of LDLCholesterol Treatment Goals in Patients With Familial Hypercholesterolemia: 5-Year SAFEHEART Registry Follow-Up. J Am Coll Cardiol. 2016; 67(11): 1278-1285, doi: 10.1016/j. jacc.2016.01.008, indexed in Pubmed: 26988947.

10. Leebmann J, Roeseler E, Julius U, et al. Lipoprotein apheresis in patients with maximally tolerated lipid-lowering therapy, lipoprotein(a)-hyperlipoproteinemia, and progressive cardiovascular disease: prospective observational multicenter study. Circulation. 2013; 128(24): 2567-2576, doi:10.1161/CIRCULATIONAHA.113.002432, indexed in Pubmed: 24056686.

11. Heigl F, Hettich R, Lotz N, et al. Clinical benefit of long-term lipoprotein apheresis in patients with severe hypercholesterolemia or $\mathrm{Lp}$ (a)-hyperlipoproteinemia with progressive cardiovascular disease. Clin Res Cardiol Suppl. 2015; 10: 8-13, doi: 10.1007/s11789-015-0071-3, indexed in Pubmed: 25672934.

12. Mabuchi H, Koizumi J, Shimizu M, et al. Long-term efficacy of low-density lipoprotein apheresis on coronary heart disease in familial hypercholesterolemia. Hokuriku-FH-LDL-Apheresis Study Group. Am J Cardiol. 1998; 82(12): 1489-1495, indexed in Pubmed: 9874053.

13. Thompson GR, Catapano A, Saheb S, et al. Severe hypercholesterolaemia: therapeutic goals and eligibility criteria for LDL apheresis in Europe. Curr Opin Lipidol. 2010; 21(6): 492-498, doi: 10.1097/MOL.0b013e3283402f53, indexed in Pubmed: 20935563.

14. Ito MK, McGowan MP, Moriarty PM. Management of familial hypercholesterolemias in adult patients: recommendations from the National Lipid Association Expert Panel on Familial Hypercholesterolemia. J Clin Lipidol. 2011; 5(3 Suppl): S38-S45, doi: 10.1016/j.jacl.2011.04.001, indexed in Pubmed: 21600528.

15. Klingel R, Fassbender T, Fassbender C, et al. From membrane differential filtration to lipidfiltration: technological progress in low-density lipoprotein apheresis. Ther Apher Dial. 2003; 7(3): 350-358, indexed in Pubmed: 12924612.

16. Stefanutti C, Mazza F, Steiner M, et al. Relationship between Sustained Reductions in Plasma Lipid and Lipoprotein Concentrations with Apheresis and Plasma Levels and mRNA Expression of PTX3 and Plasma Levels of hsCRP in Patients with HyperLp(a) lipoproteinemia. Mediators Inflamm. 2016; 2016: 4739512, doi: 10.1155/2016/4739512, indexed in Pubmed: 26903710.

17. Tamai O, Matsuoka H, Itabe H, et al. Single LDL apheresis improves endothelium-dependent vasodilatation in hypercholesterolemic humans. Circulation. 1997; 95(1): 76-82, indexed in Pubmed: 8994420.
18. Aengevaeren WR, Kroon AA, Stalenhoef AF, et al. Low density lipoprotein apheresis improves regional myocardial perfusion in patients with hypercholesterolemia and extensive coronary artery disease. LDL-Apheresis Atherosclerosis Regression Study (LAARS). J Am Coll Cardiol. 1996; 28(7): 1696-1704, indexed in Pubmed: 8962554.

19. Leebmann J, Roeseler E, Julius U, et al. Lipoprotein apheresis in patients with maximally tolerated lipid-lowering therapy, lipoprotein(a)-hyperlipoproteinemia, and progressive cardiovascular disease: prospective observational multicenter study. Circulation. 2013; 128(24): 2567-2576, doi:10.1161/CIRCULATIONAHA.113.002432, indexed in Pubmed: 24056686.

20. van Wijk DF, Sjouke B, Figueroa A, et al. Nonpharmacological lipoprotein apheresis reduces arterial inflammation in familial hypercholesterolemia. J Am Coll Cardiol. 2014; 64(14): 1418-1426, doi: 10.1016/j.jacc.2014.01.088, indexed in Pubmed: 25277610 .

21. Schettler VJJ, Neumann CL, Peter C, et al. The German Lipoprotein Apheresis Registry (GLAR) - almost 5 years on. Clin Res Cardiol Suppl. 2017; 12(Suppl 1): 44-49, doi: 10.1007/s11789017-0089-9, indexed in Pubmed: 28233268.

22. Klingel R, Heibges A, Fassbender C. Lipoprotein apheresis for Lp(a)-hyperlipoproteinemia with progressive cardiovascular disease--Additional particular aspects of the Pro(a)LiFe multicenter trial. Atheroscler Suppl. 2015; 18: 35-40, doi: 10.1016/j. atherosclerosissup.2015.02.012, indexed in Pubmed: 25936302.

23. Heigl F, Hettich R, Lotz N, et al. Efficacy, safety, and tolerability of long-term lipoprotein apheresis in patients with LDL- or Lp(a) hyperlipoproteinemia: Findings gathered from more than 36,000 treatments at one center in Germany. Atheroscler Suppl. 2015; 18: 154-162, doi:10.1016/j.atherosclerosissup.2015.02.013, indexed in Pubmed: 25936320.

24. Mickiewicz A, Chmara M, Futema M, et al. Efficacy of clinical diagnostic criteria for familial hypercholesterolemia genetic testing in Poland. Atherosclerosis. 2016; 249: 52-58, doi: 10.1016/j. atherosclerosis.2016.03.025, indexed in Pubmed: 27062410.

25. Bosch T, Schmidt B, Kleophas W, et al. LDL hemoperfusion - a new procedure for LDL apheresis: first clinical application of an LDL adsorber compatible with human whole blood. Artif Organs. 1997; 21(9): 977-982, indexed in Pubmed: 9288867.

26. Bosch T, Gahr S, Belschner U, et al. Direct adsorption of lowdensity lipoprotein by DALI-LDL-apheresis: results of a prospective long-term multicenter follow-up covering 12,291 sessions. Ther Apher Dial. 2006; 10(3): 210-218, doi: 10.1111/j.17449987.2006.00336.x, indexed in Pubmed: 16817783.

27. Kroon AA, van't Hof MA, Demacker PN, et al. The rebound of lipoproteins after LDL-apheresis. Kinetics and estimation of mean lipoprotein levels. Atherosclerosis. 2000; 152(2): 519-526, indexed in Pubmed: 10998482.

28. Lewandowski P, Romanowska-Kocejko M, Węgrzyn A, et al. Noninvasive assessment of endothelial function and vascular parameters in patients with familial and nonfamilial hypercholesterolemia. Pol Arch Med Wewn. 2014; 124(10): 516-524, indexed in Pubmed: 25187945.

29. Kroon AA, Aengevaeren WR, van der Werf T, et al. LDL-Apheresis Atherosclerosis Regression Study (LAARS). Effect of aggressive versus conventional lipid lowering treatment on coronary atherosclerosis. Circulation. 1996; 93(10): 1826-1835, indexed in Pubmed: 8635262.

30. Emmrich U, Hohenstein B, Julius U. Actual situation of lipoprotein apheresis in Saxony in 2013. Atheroscler Suppl. 2015; 18: 
215-225, doi:10.1016/j.atherosclerosissup.2015.02.034, indexed in Pubmed: 25936329.

31. Ramlow W, Röseler E, Heigl F, et al. Efficacy of lipid reduction with DALI and MONET. Atheroscler Suppl. 2017; 30: 217-224, doi:10.1016/j.atherosclerosissup.2017.05.035, indexed in Pubmed: 29096841.

32. Julius U, Fischer S, Schatz U, et al. Why an apheresis center should offer more than one lipoprotein apheresis method. Ther Apher Dial. 2013; 17(2): 179-184, doi: 10.1111/j.17449987.2012.01129.x, indexed in Pubmed: 23551674.

33. Dittrich-Riediger J, Schatz U, Hohenstein B, et al. Adverse events of lipoprotein apheresis and immunoadsorption at the Apheresis Center at the University Hospital Dresden. Atheroscler Suppl. 2015; 18: 45-52, doi: 10.1016/j.atherosclerosissup.2015.02.007, indexed in Pubmed: 25936304.

34. Koziolek MJ, Hennig U, Zapf A, et al. Retrospective analysis of long-term lipid apheresis at a single center. Ther Apher Dial. 2010; 14(2): 143-152, doi:10.1111/j.1744-9987.2009.00747.x, indexed in Pubmed: 20438535.

35. Kozik-Jaromin J, Röseler E, Heigl F, et al. Safety aspects of lipidapheresis using DALI and MONET — Multicenter observational study. Atheroscler Suppl. 2017; 30: 225-231, doi: 10.1016/j. atherosclerosissup.2017.05.036, indexed in Pubmed: 29096842.

36. Kalantari K. The choice of vascular access for therapeutic apheresis. J Clin Apher. 2012; 27(3): 153-159, doi: 10.1002/jca.21225, indexed in Pubmed:22535654.

37. Doherty DJ, Pottle A, Malietzis G, et al. Vascular access in lipoprotein apheresis: a retrospective analysis from the UK's largest lipoprotein apheresis centre. J Vasc Access. 2018; 19(1): 52-57, doi: 10.5301/jva.5000755, indexed in Pubmed: 29076516.

38. Leebmann J, Roeseler E, Julius U, et al. Lipoprotein apheresis in patients with maximally tolerated lipid-lowering therapy, lipoprotein(a)-hyperlipoproteinemia, and progressive cardiovascular disease: prospective observational multicenter study. Circulation. 2013; 128(24): 2567-2576, doi:10.1161/CIRCULATIONAHA.113.002432, indexed in Pubmed: 24056686.

39. Sampietro T, Sbrana F, Bigazzi F, et al. The incidence of cardiovascular events is largely reduced in patients with maximally tolerated drug therapy and lipoprotein apheresis. A single-center experience. Atheroscler Suppl. 2015; 18: 268-272, doi: 10.1016/j.atherosclerosissup.2015.02.040, indexed in Pubmed: 25936336.

40. Saborowski M, Dölle M, Manns MP, et al. Lipid-lowering therapy with PCSK9-inhibitors in the management of cardiovascular high-risk patients: Effectiveness, therapy adherence and safety in a real world cohort. Cardiol J. 2018; 25(1): 32-41, doi: 10.5603/ CJ.a2017.0137, indexed in Pubmed:29168543.

41. Kapłon-Cieślicka A, Michalak M, Kołtowski Ł, et al. How has the treatment of hypercholesterolemia in Poland changed over the last six years? Cardiol J. 2017; 24(3): 266-275, doi: 10.5603/ CJ.a2017.0047, indexed in Pubmed: 28394011.

42. Vishwanath R, Hemphill LC. Familial hypercholesterolemia and estimation of US patients eligible for low-density lipoprotein apheresis after maximally tolerated lipid-lowering therapy. J Clin Lipidol. 2014; 8(1): 18-28, doi: 10.1016/j.jacl.2013.11.002, indexed in Pubmed: 24528684. 